\title{
Hepatitis C Virus Core Protein Suppresses Mitophagy by Interacting with Parkin in the Context of Mitochondrial Depolarization
}

Yuichi Hara, ${ }^{*}$ Izumi Yanatori, ${ }^{\dagger}$ Masanori Ikeda, ${ }^{\ddagger}$ Emi Kiyokage, ${ }^{\S}$ Sohji Nishina, ${ }^{*}$ Yasuyuki Tomiyama, ${ }^{*}$ Kazunori Toida, Fumio Kishi, ${ }^{\dagger}$ Nobuyuki Kato, Michio Imamura, "Kazuaki Chayama, ${ }^{\ddagger}$ and Keisuke Hino*

From the Departments of Hepatology and Pancreatology, ${ }^{*}$ Molecular Genetics, ${ }^{\dagger}$ and Anatomy, ${ }^{\S}$ Kawasaki Medical School, Kurashiki; the Department of Tumor Virology, ${ }^{\ddagger}$ Okayama University Graduate School of Medicine, Dentistry and Pharmaceutical Sciences, Okayama; and the Department of Gastroenterology and Metabolism, "Applied Life Sciences, Institute of Biomedical and Health Sciences, Hiroshima University, Hiroshima, Japan

Accepted for publication

July $25,2014$.

Address correspondence to Keisuke Hino, M.D., Ph.D., Department of Hepatology and Pancreatology, Kawasaki Medical School, 577 Matsushima, Kurashiki, Okayama 701-0192, Japan. E-mail: khino@med. kawasaki-m.ac.jp.
Hepatitis $\mathrm{C}$ virus ( $\mathrm{HCV}$ ) causes mitochondrial injury and oxidative stress, and impaired mitochondria are selectively eliminated through autophagy-dependent degradation (mitophagy). We investigated whether HCV affects mitophagy in terms of mitochondrial quality control. The effect of HCV on mitophagy was examined using HCV-Japanese fulminant hepatitis-1-infected cells and the uncoupling reagent carbonyl cyanide $m$-chlorophenylhydrazone as a mitophagy inducer. In addition, liver cells from transgenic mice expressing the HCV polyprotein and human hepatocyte chimeric mice were examined for mitophagy. Translocation of the E3 ubiquitin ligase Parkin to the mitochondria was impaired without a reduction of pentaerythritol tetranitrate-induced kinase 1 activity in the presence of HCV infection both in vitro and in vivo. Coimmunoprecipitation assays revealed that Parkin associated with the HCV core protein. Furthermore, a Yeast Two-Hybrid assay identified a specific interaction between the HCV core protein and an N-terminal Parkin fragment. Silencing Parkin suppressed HCV core protein expression, suggesting a functional role for the interaction between the HCV core protein and Parkin in HCV propagation. The suppressed Parkin translocation to the mitochondria inhibited mitochondrial ubiquitination, decreased the number of mitochondria sequestered in isolation membranes, and reduced autophagic degradation activity. Through a direct interaction with Parkin, the HCV core protein suppressed mitophagy by inhibiting Parkin translocation to the mitochondria. This inhibition may amplify and sustain HCV-induced mitochondrial injury. (Am J Pathol 2014, 184: 3026-3039; http:/) dx.doi.org/10.1016/j.ajpath.2014.07.024)
Oxidative stress is present in chronic hepatitis $\mathrm{C}$ to a greater degree than in other inflammatory liver diseases. ${ }^{1,2}$ The hepatitis $\mathrm{C}$ virus $(\mathrm{HCV})$ core protein induces the production of reactive oxygen species (ROS) ${ }^{3,4}$ through mitochondrial electron transport inhibition. ${ }^{5}$ Because the mitochondria are targets for ROS and ROS generators, $\mathrm{HCV}$-induced ROS have the potential to injure mitochondria. In addition, hepatocellular mitochondrial alterations have been observed in patients with chronic hepatitis $\mathrm{C}^{6}{ }^{6} \mathrm{We}$ previously identified a ROS-associated iron metabolic disorder ${ }^{7}$ and demonstrated that transgenic mice expressing the $\mathrm{HCV}$ polyprotein develop hepatocarcinogenesis related to mitochondrial injury induced by HCV and iron overload. ${ }^{8}$ Therefore, impaired mitochondrial function may play a critical role in the development of hepatocellular carcinoma (HCC) in patients with chronic $\mathrm{HCV}$ infection. Conversely, the affected mitochondria are selectively eliminated through the autophagy-dependent degradation of mitochondria (referred to as mitophagy) in both physiological and pathological settings to maintain the mitochondrial quality. ${ }^{9,10}$ On the

\footnotetext{
Supported by Japan Society for the Promotion of Science Grant-in-Aid for Scientific Research (B) 23390201 and Grant-in-Aid for Exploratory Research 25670374; Ministry of Health, Labor and Welfare of Japan Health and Labor Sciences Research grant 25200601 for research on hepatitis; and Kawasaki Medical School Research Project grant P2.

Disclosures: None declared.

Current address of M.I., Kagoshima University Graduate School of Medical and Dental Sciences, Kagoshima, Japan.
} 
basis of these observations, we hypothesized that HCV may suppress mitophagy, which could lead to the sustained presence of affected mitochondria, increased ROS production, and the development of HCC.

Mitochondrial membrane depolarization precedes mitophagy induction, ${ }^{11}$ which is selectively controlled by a variety of proteins in mammalian cells, including pentaerythritol tetranitrate-induced kinase 1 (PINK1) and the E3 ubiquitin ligase Parkin. ${ }^{12-19}$ PINK1 facilitates Parkin targeting of the depolarized mitochondria. ${ }^{12-15}$ Although Parkin ubiquitinates a broad range of mitochondrial outer membrane proteins, ${ }^{14,17-19}$ it remains unclear how Parkin enables the damaged mitochondria to be recognized by the autophagosome. Structures containing autophagy-related protein 9A and the uncoordinated family member-51-like kinase 1 complex independently target depolarized mitochondria at the initial stages of Parkin-mediated mitophagy, whereas the autophagosomal microtubule-associated protein light chain 3 (LC3) is critical for efficient incorporation of damaged mitochondria into the autophagosome at a later stage. ${ }^{20}$ LC3-I undergoes post-translational modification by phosphatidylethanolamine to become LC3-II, and LC3-II insertion into the autophagosomal membrane is a key step in autophagosome formation. In addition, the autophagic adaptor p62 is recruited to mitochondrial clusters and is essential for mitochondrial clearance, ${ }^{13}$ although it remains controversial as to whether p62 is essential for mitochondrial recognition by the autophagosome ${ }^{13}$ or rather is important for perinuclear clustering of depolarized mitochondria. ${ }^{19,21}$ Our aim was to examine whether HCV suppresses mitophagy. We found that HCV core protein inhibits the translocation of Parkin to affected mitochondria by interacting with Parkin and subsequently suppressing mitophagy. These results imply that mitochondria affected by HCV core protein are unlikely to be eliminated, which may intensify oxidative stress and increase the risk of hepatocarcinogenesis.

\section{Materials and Methods}

\section{Cell Culture, HCV Infection Experiments, and Mitochondrial Depolarization}

HCV-Japanese fulminant hepatitis-1 (JFH1)-infected Huh7 cells have previously been described in detail. ${ }^{22}$ The supernatants were collected from cell culture-generated JFH1-Huh7 cells at 21 days after infection and stored until use at $-80^{\circ} \mathrm{C}$ after filtering through a $0.45-\mu \mathrm{m}$ filter. For infection experiments with the HCV-JFH1 virus, $1 \times 10^{5}$ Huh7 cells per well were plated onto 6-well plates and cultured for 24 hours. Then, we infected the cells with $50 \mu \mathrm{L}$ (equivalent to a multiplicity of infection of 0.1) of inoculum. The culture supernatants were collected, and the levels of the HCV core were determined using an enzyme-linked immunosorbent assay (ELISA; Mitsubishi Kagaku Bio-Clinical Laboratories, Tokyo, Japan). Total RNA was isolated from the infected cellular lysates using an RNeasy mini kit (Qiagen, Hilden, Germany) for quantitative
RT-PCR analysis of the intracellular HCV RNA. The HCV infectivity in the culture supernatants was determined by a focus-forming assay at 48 hours after infection. The HCVinfected cells were detected using an anti-HCV core antibody (CP-9 and CP-11, Institute of Immunology, Ltd, Tokyo, Japan). Intracellular HCV infectivity was determined using a focus-forming assay at 48 hours after inoculation of the lysates by repeated freeze-and-thaw cycles (three times).

To depolarize the mitochondria, the cells were treated with $10 \mu \mathrm{mol} / \mathrm{L}$ carbonyl cyanide $m$-chlorophenylhydrazone (CCCP; Sigma-Aldrich, St. Louis, MO) for 1 to 2 hours or 1 $\mu \mathrm{mol} / \mathrm{L}$ valinomycin (Sigma-Aldrich) for 3 hours; CCCP represses ATP synthesis through the loss of the $\mathrm{H}^{+}$gradient without affecting mitochondrial electron transport, which is known to induce mitochondrial fragmentation. ${ }^{13}$

\section{Animals}

The pAlbSVPA-HCV vector, which contains the full-length polyprotein-coding region under the control of the murine albumin promoter/enhancer, has previously been described in detail. ${ }^{23,24}$ Of the four transgenic lineages with evidence of RNA transcription of the full-length HCV-N open reading frame (FL-N), the FL-N/35 lineage proved capable of breeding large numbers. ${ }^{24}$ Urokinase-type plasminogen activator-transgenic severe combined immunodeficiency mice were generated, and human hepatocytes were transplanted to generate chimeric mice. ${ }^{25}$ The chimeric mice were injected with genotype $1 b \mathrm{HCV}$-positive human serum samples, as described previously. ${ }^{26}$ The mouse livers were extracted 12 weeks after the infection, when the serum HCV RNA titers had increased over baseline levels. Male FL-N/35 transgenic mice, age-matched C57BL/6 mice (control), and chimeric mice with and without HCV infection were fed, maintained, and then euthanized by i.p. injection of $10 \%$ nembutal sodium, according to the guidelines approved by the Institutional Animal Care and Use Committee. The study protocol for obtaining human serum samples conformed to the ethical guidelines of the 1975 Declaration of Helsinki and was approved by the Institutional Review Committee.

\section{Measurement of HCV RNA and Human Albumin in the} Serum of Chimeric Mice

HCV RNA ${ }^{26}$ and human albumin ${ }^{25}$ were quantified as described previously. Human albumin levels in the serum of chimeric mice were determined using the Human Albumin ELISA Quantification kit (Bethyl Laboratories Inc., Montgomery, TX).

\section{Measurement of Mitochondrial Membrane Potential}

The mitochondrial membrane potential $(\Delta \Psi)$ was measured using a Cell Meter JC-10 Mitochondrial Membrane Potential Assay kit (AAT Bioquest, Inc., Sunnyvale, CA), according to the manufacturer's instructions. The fluorescent intensities 
for both J-aggregates (red) and monometric forms (green) of JC-10 were measured at Ex/Em $=490 / 525 \mathrm{~nm}$ and 540/590 nm with a Varioskan Flush Multimode Reader (Thermo Fisher Scientific, Waltham, MA).

\section{Isolation of Mitochondria}

The cells were lysed by mechanical homogenization using a small pestle, and mitochondrial extraction was performed using a Qproteome Mitochondria Isolation kit (Qiagen), according to the manufacturer's instructions. Liver mitochondria were isolated as described previously with some modifications. ${ }^{27}$ In brief, the livers were minced on ice and homogenized by five strokes with a Dounce homogenizer and a tight-fitting pestle in isolation buffer $\left[70 \mathrm{mmol} / \mathrm{L}\right.$ sucrose, $1 \mathrm{mmol} / \mathrm{L} \mathrm{KH}_{2} \mathrm{PO}_{4}, 5$ $\mathrm{mmol} / \mathrm{L}$ HEPES, $220 \mathrm{mmol} / \mathrm{L}$ mannitol, $5 \mathrm{mmol} / \mathrm{L}$ sodium succinate, and $0.1 \%$ bovine serum albumin (BSA), $\mathrm{pH}$ 7.4]. The homogenate was centrifuged at $800 \times g$ for 5 minutes at $4^{\circ} \mathrm{C}$. The supernatant fraction was retained, whereas the pellet was washed with isolation buffer and centrifuged again. The combined supernatant fractions were centrifuged at $1000 \times g$ for 15 minutes at $4^{\circ} \mathrm{C}$ to obtain a crude mitochondrial pellet.

\section{Measurement of ROS}

The cellular ROS level was measured by oxidation of the cellpermeable, oxidation-sensitive fluorogenic precursor, $2^{\prime}, 7^{\prime}$ dihydrodichlorofluorescein diacetate (Molecular Probes Inc., Eugene, OR). Fluorescence was measured using a Varioskan Flush Multimode Reader at 495/535 nm (excitation/emission).

\section{Determination of Glutathione Content}

Mitochondrial pellets were measured for total glutathione [reduced glutathione $(\mathrm{GSH})+$ oxidized glutathione (GSSG)] and GSH content using the GSSG/GSH Quantification kit (Dojindo Molecular Technologies, Inc., Kumamoto, Japan). The concentration of GSH was calculated using the following formula:

$$
\begin{aligned}
& \text { GSH concentration }=\text { Total glutathione concentration } \\
& -[\text { GSSG concentration }] \times 2
\end{aligned}
$$

The liver tissue samples (approximately $50 \mathrm{mg}$ ) were minced in ice-cold metaphosphoric acid solution, homogenized, and centrifuged at $3000 \times g$ for 10 minutes at $4^{\circ} \mathrm{C}$. Lysates from the liver tissue samples and mitochondrial samples $(2 \mathrm{mg})$ were evaluated for the concentration of GSH using the thioester method and a GSH-400 kit (Oxis International Inc., Portland, $\mathrm{OR}$ ) and for total glutathione content using the glutathione reductase-dinitrothiocyanobenzene recycling assay and the GSH-412 kit (Oxis International Inc.), as described previously. ${ }^{5}$

\section{Immunoblotting}

Samples were lysed in radioimmunoprecipitation assay buffer [20 mmol/L Tris-HCl (pH 7.5), $150 \mathrm{mmol} / \mathrm{L} \mathrm{NaCl}$,
$50 \mathrm{mmol} / \mathrm{L} \mathrm{NaF}, 1 \mathrm{mmol} / \mathrm{L} \mathrm{Na} \mathrm{VO}_{4}, 0.1 \%$ SDS, and $0.5 \%$ Triton X-100], as described previously, ${ }^{28}$ supplemented with $1 \%$ protease inhibitor mixture (Sigma-Aldrich) and 100 $\mathrm{mmol} / \mathrm{L}$ phenylmethylsulfonyl fluoride. Cell lysates or mitochondrial pellets were subjected to immunoblot analysis using an iBlot Gel Transfer Device (Invitrogen, Carlsbad, CA). The membranes were incubated with the following primary antibodies: rabbit anti-human LC3 (Novus Biologicals, Littleton, CO), rabbit anti-human p62/ SQSTM1 (MBL, Nagoya, Japan), rabbit anti-human Parkin (Cell Signaling Technology, Danvers, MA), mouse antihuman Parkin (Santa Cruz Biotechnology, Inc.), rabbit anti-human p-Parkin (Ser 378; Santa Cruz Biotechnology, Inc.), rabbit anti-human PINK1 (Cell Signaling Technology), mouse anti-human mitochondrial heat shock protein-70 (BioReagents, Golden, CO), mouse anti-human ubiquitin (Santa Cruz Biotechnology, Inc.), goat anti-human voltagedependent anion-selective channel protein 1 (VDAC1; Santa Cruz Biotechnology, Inc.), monoclonal antisynthetic HCV core peptide (CP11; Institute of Immunology, Ltd), mouse anti-HCV non-structural (NS) 3 protein (Abcam, Cambridge, MA), mouse anti-HCV NS4A (Abcam), mouse anti-HCV NS5A protein (Abcam), and rabbit anti-human $\beta$-actin (Cell Signaling Technology).

\section{Electron Microscopy}

To address the detail localization of core and Parkin, the cells treated with CCCP for 1 hour were fixed with $4 \%$ paraformaldehyde and $1 \%$ glutaraldehyde in $0.1 \mathrm{~mol} / \mathrm{L}$ Millonig's phosphate buffer $(\mathrm{pH}$ 7.4) for 30 minutes. The cells were incubated with a mixture of the following primary antibodies in phosphate-buffered saline (PBS) containing $1 \% \mathrm{BSA}$ and $0.05 \%$ sodium azide overnight at $20^{\circ} \mathrm{C}$ : mouse monoclonal antisynthetic HCV core peptide (Institute of Immunology), rabbit anti-human Parkin (Abcam), and rabbit anti-rat LC3 (Wako Pure Chemical Industries, Ltd, Osaka, Japan). After washing with PBS, the cells were incubated with biotinylated donkey anti-rabbit IgG (Jackson ImmunoResearch Laboratories, Inc., Baltimore Pike, PA) in $1 \%$ BSA for 2 hours at $20^{\circ} \mathrm{C}$. After washing with PBS, the cells were incubated with Alexa Fluor-488 FluoroNanogold-streptavidin (Jackson ImmunoResearch Laboratories, Inc.), indocarbocyanine-labeled donkey anti-mouse IgG (Jackson ImmunoResearch Laboratories, Inc.), and indocarbocyanine-labeled donkey anti-rabbit IgG (Jackson ImmunoResearch Laboratories, Inc.) in 1\% BSA for 2 hours at $20^{\circ} \mathrm{C}$. After washing with PBS, the cells were incubated with mouse peroxidase-anti-peroxidase complex (Jackson ImmunoResearch Laboratories, Inc.) in PBS for 3 hours at $20^{\circ} \mathrm{C}$. The peroxidase reduction was developed with $0.05 \%$ diaminobenzidine tetrahydrochloride in $50 \mathrm{mmol} / \mathrm{L}$ Tris buffer containing $0.01 \%$ hydrogen peroxide for 20 minutes at room temperature. The diameter of the gold immunoparticles was increased using a silver enhancement kit (HQ silver; Nanoprobes, Inc., Yaphank, NY) for 4 minutes at 
room temperature. After treatment with $1 \%$ osmium and $2 \%$ uranyl acetate, the cells were dehydrated in a graded series of ethanol and embedded in Epon-Araldite (OKEN, Tokyo, Japan). Serial ultrathin sections (each $70 \mathrm{~nm}$ thick) were examined using an electron microscope (model JEM1400; JEOL, Tokyo, Japan). These immune-electron microscopic methods were generally performed according to our previous study. ${ }^{29}$

\section{Immunofluorescence Microscopy}

The cells were fixed, permeabilized, and immunostained with rabbit anti-human Parkin (Abcam), goat anti-human Parkin (Santa Cruz Biotechnology, Inc.), goat anti-human Tom20 (Santa Cruz Biotechnology, Inc.), rabbit anti-rat LC3 (Wako Pure Chemical Industries, Ltd), or mouse monoclonal antisynthetic HCV core peptide (Institute of Immunology) antibodies, followed by Cy3-conjugated donkey anti-rabbit IgG (Jackson ImmunoResearch Laboratories, Inc.), fluorescein isothiocyanate-conjugated donkey anti-goat IgG (Jackson ImmunoResearch Laboratories, Inc.), or Alexa Fluor 647-conjugated donkey anti-mouse IgG (Jackson ImmunoResearch Laboratories, Inc.). Cell images were captured using a confocal microscope (model LSM700; Zeiss, Jena, Germany) equipped with 488-, 555-, and 639-nm diodes. The images were acquired in a sequential mode using a $63 \times$ Plan Apochromat numerical aperture/1.4 oil objective and the appropriate filter combinations. All images were saved as tagged image file format files. The contrast was adjusted using Photoshop version CS5 (Adobe, San Jose, CA), and the images were imported into Illustrator version CS5 (Adobe). Colocalization was assessed with line scans using ImageJ software version $1.46(\mathrm{NIH}$, Bethesda, MD).

\section{Coimmunoprecipitation}

Coimmunoprecipitation was performed using a Dynabeads CoImmunoprecipitation Kit (Invitrogen), according to the manufacturer's instructions. Magnetic beads (Dynabeads M-270 Epoxy) were conjugated to anti-VDAC1 (Santa Cruz Biotechnology, Inc.), anti-Parkin (Cell Signaling Technology), antiubiquitin (Santa Cruz Biotechnology, Inc.), or anti-p62 (MBL) antibodies by rotating overnight at $37^{\circ} \mathrm{C}$. The antibodyDynabeads complex was then treated with coupling buffer. Beads coupled to anti-VDAC, anti-Parkin, anti-ubiquitin, or anti-p62 were incubated with cell lysates for 30 minutes at $4{ }^{\circ} \mathrm{C}$ and then washed with coupling buffer. Collected protein complexes were subjected to immunoblot analysis using antiVDAC, anti-ubiquitin (Santa Cruz Biotechnology, Inc.), and anti-Parkin (Cell Signaling Technology) antibodies to detect coimmunoprecipitated VDAC1, ubiquitin, and Parkin. Immunoblots using anti-Parkin, anti-HCV core (Institute of Immunology), anti-HCV NS3 (Abcam), anti-HCV NS4A (Abcam), or anti-HCV NS5A (Abcam) antibodies were performed to detect the coimmunoprecipitation of Parkin with core, NS3, NS4A, or NS5A protein.

\section{RNA Interference}

The siRNA knockdown oligonucleotides were obtained from Invitrogen. JFH1-Huh7 cells and/or Huh7 cells were grown to $50 \%$ to $60 \%$ confluency and transfected with 100 pmol siRNA oligonucleotides [5'-GGACGCUGUUCCUCGUUAUGAAGAA-3' (forward) and 5'-UUCUUCAUAACGAGGAACAGCGUCC-3' (reverse)] for PINK1 or siRNA oligonucleotides [5'-UCCAGCUCAAGGAGGUGGUUGCUAA-3' (forward) and 5'-UUAGCAACCACCUCCUUGAGCUGGA-3' (reverse)] for Parkin using Lipofectamine 2000 (Invitrogen). The cells were analyzed 72 hours after transfection.

\section{Yeast Two-Hybrid Assay}

A Matchmaker Gal4 two-hybrid system 3 (Clontech Laboratories, Inc., Mountain View, CA) was used according to the manufacturer's instructions. Saccharomyces cerevisiae Y187, containing an $\mathrm{N}$ - or C-terminal fragment cDNA of Parkin as a prey cloned into the Gal4-activation domain vector (pACT2), was allowed to mate with $S$. cerevisiae AH109, which had been transformed with a Gal4 DNAbinding domain vector (pGBKT7) containing the HCV core as bait. In addition, S. cerevisiae Y187, with the HCV core as a prey cloned into the Gal4-activation domain vector (pACT2), was allowed to mate with S. cerevisiae AH109, which had been transformed with a Gal4 DNA-binding domain vector (pGBKT7) containing $\mathrm{N}$ - or C-terminal fragment cDNA of Parkin as bait. To construct the prey and the bait, two regions of the Parkin gene that encoded the Nterminal 215-amino acid residues (1 to 215) and the Cterminal 250-amino acid residues (216 to 465) were amplified using PCR with genomic cDNA, and the HCV core gene was amplified with the HCV-O (genotype $1 b$ ) genomic cDNA. ${ }^{30}$ The PCR primers were as follows with the incorporated BamHI and EcoRI sites underlined: Parkin 1 to $215,5^{\prime}$-GGATCCGCATGATAGTGTTTGTCAGGTT-3' (forward) and 5'-GAATTCCTAGTGTGCTCCACATTTAAAGA-3' (reverse); Parkin 216 to 465, 5'-GGATCCGCCCCACCTCTGACAAGGAAAC-3' (forward) and 5'-GAATTCCTACACGTCGAACCAGTGGT-3' (reverse); and HCV core, 5'-GAATTCGCCATGAGCACAAATCCTAAACCTC-3' (forward) and $5^{\prime}$-GGATCCTTAAGCGGAAGCTGGGATGGTCAAA-3' (reverse).

\section{Real-Time RT-PCR}

Total RNA was extracted from frozen liver tissues and cells using the RNeasy mini kit (Qiagen). Total RNA $(2 \mu \mathrm{g})$ was reverse transcribed to cDNA using the High-Capacity RNA to cDNA kit (Applied Biosystems, Foster City, CA), according to the manufacturer's instructions. TaqMan Gene Expression Assays for LC3B, glyceraldehyde-3-phosphate dehydrogenase (GAPDH), Parkin, and HCV core were purchased from Applied Biosystems, and mRNA levels were quantified in triplicate using an Applied Biosystems 
7500 Real-Time PCR system, according to the supplier's recommendations. The expression value for LC3B, Parkin, and $\mathrm{HCV}$ core mRNA was normalized to that of GAPDH.

\section{Statistical Analysis}

Quantitative values are expressed as the means \pm SD. Data were compared between the two groups using the Student's $t$-test. $P<0.05$ was considered significant.

\section{Results}

\section{Mitochondrial Oxidative Status in Vitro and in Vivo}

After treatment with CCCP, a widely adopted reagent for inducing mitophagy, the mitochondrial membrane potential $(\Delta \Psi)$ was significantly reduced irrespective of HCV infection (Figure 1A). The ratio of reduced/total glutathione content was decreased in the mitochondrial fraction after CCCP treatment in JFH1-Huh7 cells (Figure 1B). Thus, the mitochondrial oxidative status after CCCP treatment was present in HCV-infected cells (JFH1-Huh7). The ratio of reduced/total glutathione content was also decreased in the mitochondrial fraction but not in the whole liver in transgenic mice and $\mathrm{HCV}$-infected chimeric mice compared with the control mice (Figure 1, C and D). These results suggest that there is a baseline oxidation level within the mitochondrial glutathione pool in these transgenic mice and $\mathrm{HCV}$-infected chimeric mice. Furthermore, the mitochondria in these transgenic mice and $\mathrm{HCV}$-infected chimeric mice can undergo mitophagy.

\section{Impaired Recruitment of Parkin to the Mitochondria}

Parkin phosphorylation and translocation to the mitochondria after CCCP treatment are indispensable for mitochondrial ubiquitination and subsequent autophagosome formation during the course of mitophagy. ${ }^{13,15} \mathrm{CCCP}$ exposure induced Parkin accumulation in the mitochondria of Huh7 cells; however, this Parkin recruitment seemed to be inhibited in JFH1-Huh7 cells (Figure 2A). CCCP treatment induces mitochondrial fission, followed by mitophagy. ${ }^{13}$ CCCPtreated Huh7 cells displayed fragmented mitochondria colocalized with Parkin, except for a few mitochondrial tubular network cells. Western blot analysis also showed that CCCP-induced recruitment of Parkin to the mitochondria was suppressed without any change in Parkin expression or phosphorylation levels in whole cell lysates of JFH1-Huh7 cells (Figure 2B). Neither CCCP treatment nor HCV infection significantly increased the mRNA levels of Parkin in Huh7 cells, even though there was a tendency of increase in Parkin mRNA after HCV infection (Figure 2C). These results indicate that $\mathrm{HCV}$ infection could inhibit Parkin recruitment to CCCP-induced depolarized mitochondria.

The unique and high concentration of CCCP $(10 \mu \mathrm{mol} / \mathrm{L})$ used in the present study may have affected cellular functions
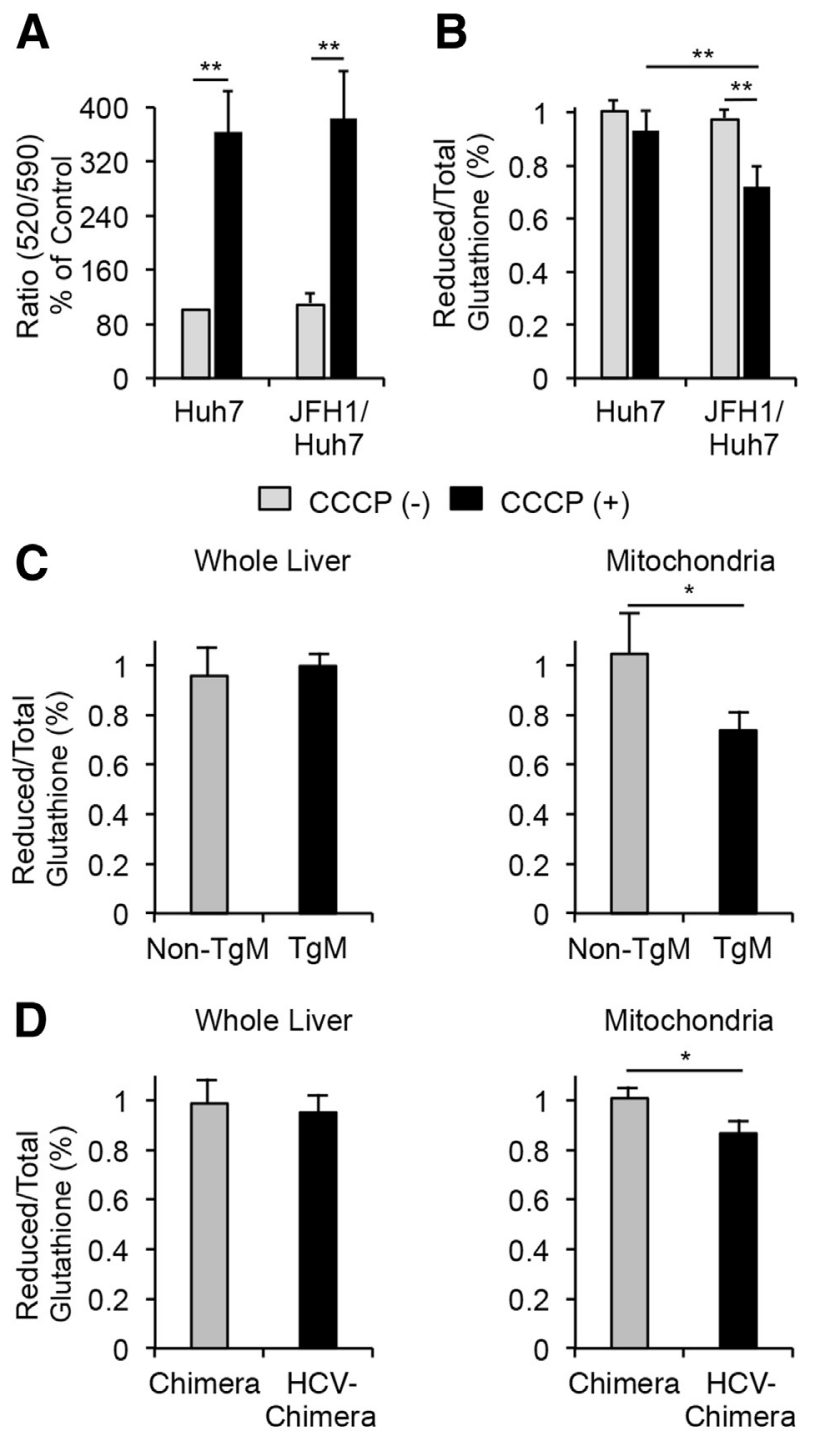

Figure 1 Mitochondrial membrane potential $(\Delta \Psi)$ and glutathione content. A: Changes in $\Delta \Psi$ levels after a 1-hour carbonyl cyanide $m$-chlorophenylhydrazone (CCCP) treatment for Huh7 and JFH1-Huh7 cells $(n=5)$. The $y$ axis represents the ratio of red (JC-10 aggregate form)/ green (JC-10 monometric form) fluorescence intensity. B: Reduced and total glutathione content in mitochondrial fractions $(n=5)$. Reduced glutathione content was normalized to total glutathione content. Reduced and total glutathione content of freshly isolated whole liver homogenates or mitochondrial fractions of transgenic livers $(n=7, \mathrm{C})$ or HCV-infected chimeric mice livers $(n=5, \mathbf{D})$ compared with the content in the corresponding control liver samples. Reduced glutathione content was normalized to total glutathione content. ${ }^{*} P<0.05,{ }^{*} P P<0.01$.

other than the proton gradient, ${ }^{31}$ which suggests that Parkin translocation from the cytoplasm to the mitochondria may not be induced specifically through mitochondrial depolarization. Therefore, we examined mitochondrial accumulation of Parkin using lower CCCP concentrations $(0.1,1,5$, or 10 $\mu \mathrm{mol} / \mathrm{L})$. In coimmunoprecipitation experiments, CCCP exposure induced ubiquitinated Parkin accumulation in the mitochondria in a dose-dependent manner in Huh7 cells, as described previously, ${ }^{13}$ but did not induce these changes in JFH1-Huh7 cells (Figure 2D). These results suggest that 

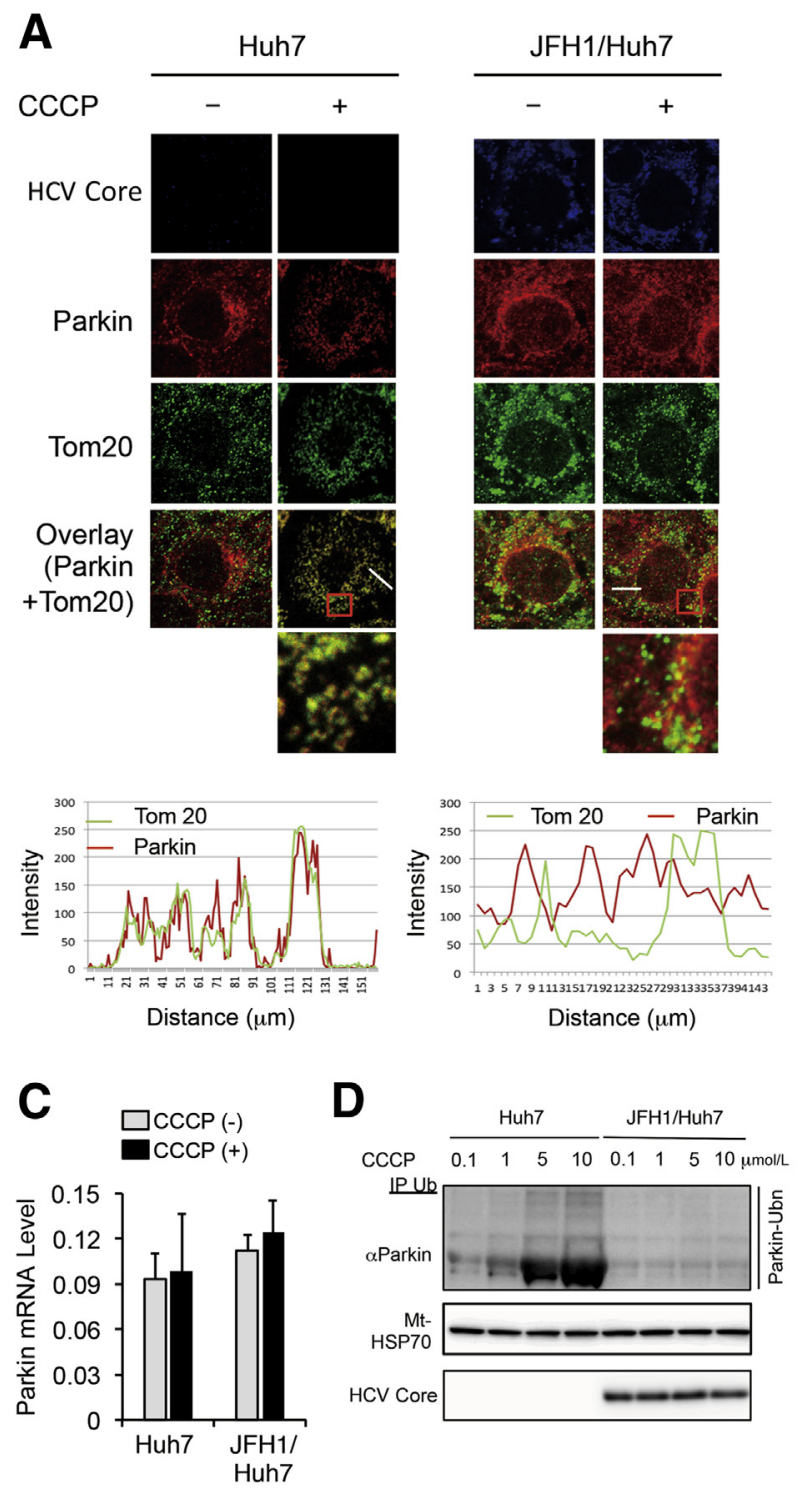
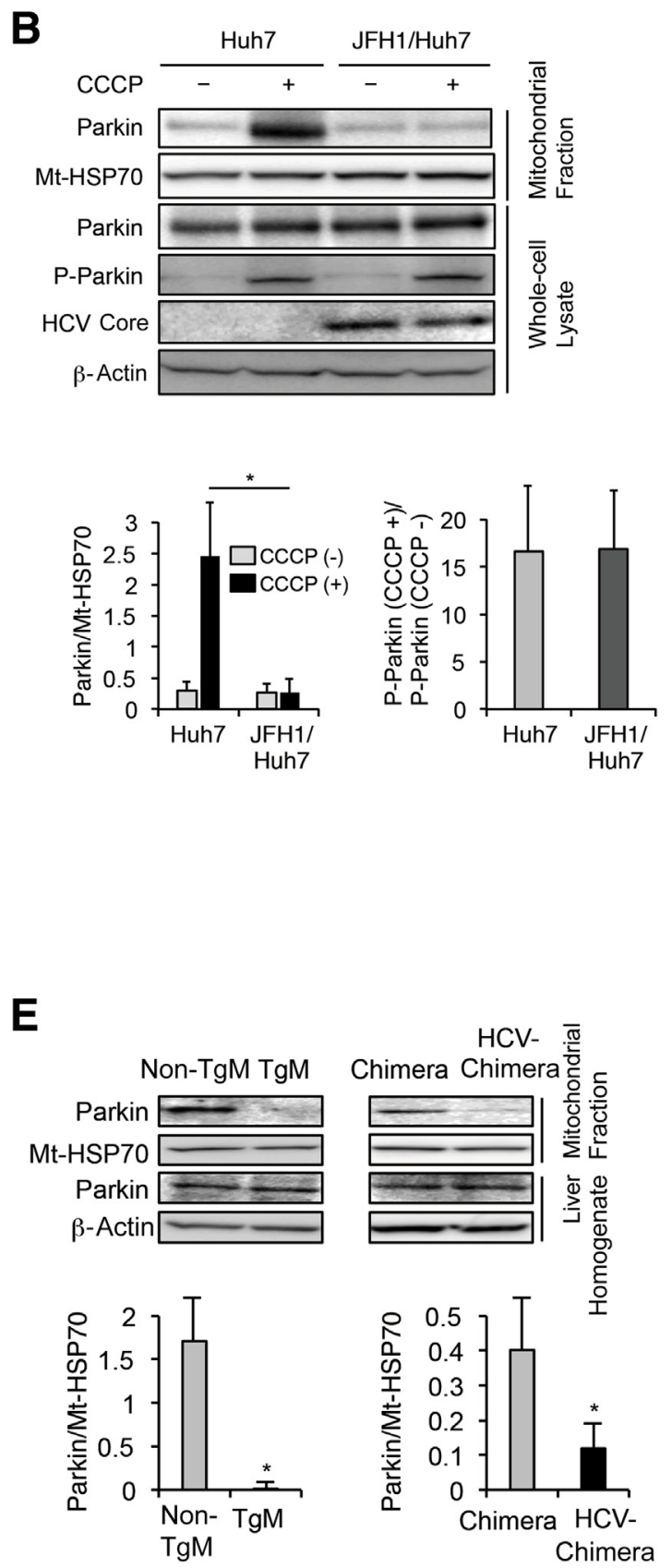

Figure 2 Effect of HCV on the translocation of Parkin to the mitochondria. A: Immunofluorescence staining for Parkin (red) and the mitochondrial marker Tom20 (green) in Huh7 and JFH1-Huh7 cells before $(-)$ and after $(+)$ carbonyl cyanide $m$-chlorophenylhydrazone (CCCP) treatment for 1 hour. Boxed areas are enlarged below. Endogenous Parkin that colocalizes with the mitochondria (yellow spots). Line scans indicate the colocalization of Parkin with the mitochondria and correlate to the white lines in the images. Boxed areas are enlarged below. B: Immunoblots for Parkin and phosphorylated Parkin ( $\mathrm{p}$-Parkin) using the mitochondrial fractions and whole cell lysates before and after CCCP treatment $(n=5)$. Parkin expression level was normalized to mitochondrial heat shock protein 70 (Mt-HSP70). The degree of phosphorylation was expressed as the ratio of phosphorylated Parkin after CCCP treatment to that prior treatment. C: Parkin mRNA level in Huh7 cells and JFH1-Huh7 cells before and after CCCP treatment $(n=5)$. The expression level for Parkin was normalized to GAPDH. D: Coimmunoprecipitation reveals more ubiquitinated Parkin in CCCP dose-dependent manner in Huh7 cells but not in JFH1-Huh7 cells. E: Immunoblots for Parkin using mitochondrial fractions of the livers or liver homogenates from non-TgM and TgM and from chimeric mice with or without HCV infection ( $n=5$ for each type of mouse). ${ }^{*} P<0.05$.

CCCP specifically induces mitophagy in Huh7 cells and that the HCV infection has an inhibitory effect on mitophagy in JFH1-Huh7 cells.

FL-N/35-transgenic mice and HCV-infected chimeric mice also showed reduced Parkin expression in the mitochondrial fraction of the liver with no change in Parkin expression levels in whole liver homogenates (Figure 2E). Serum human albumin levels, which serve as useful markers for the extent of replacement with human hepatocytes, were $16.0 \pm 7.2 \mathrm{mg} / \mathrm{mL}$ in chimeric mice with $\mathrm{HCV}$ infection and $11.9 \pm 1.7 \mathrm{mg} / \mathrm{mL}$ in chimeric mice without $\mathrm{HCV}$ infection (Figure $3 \mathrm{~A}$ ). These findings suggest that there was 


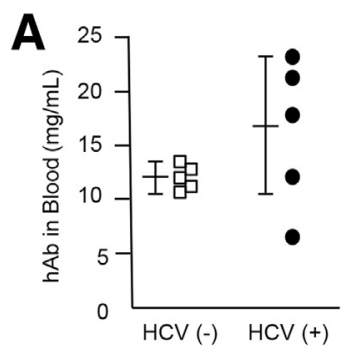

B

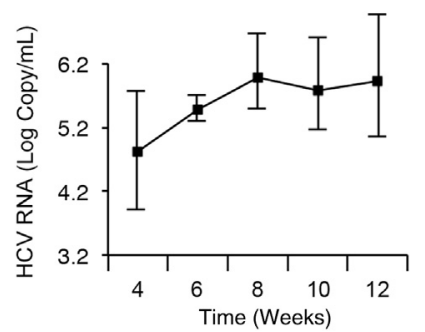

Figure 3 Human albumin and HCV RNA levels in the serum of chimeric mice with or without HCV infection. A: Human albumin (hAb) levels in the serum of 3-month-old chimeric mice with or without HCV infection. B: Serial change in HCV RNA levels in the serum after HCV infection in chimeric mice $(n=5)$.

a replacement index of $>90 \%$ according to a graph of the correlation between these two parameters identified in a previous study. ${ }^{25}$ Moreover, serum HCV RNA levels increased after infection with HCV (Figure 3B). HCV infection also suppressed the translocation of Parkin to the mitochondria in human hepatocytes.

\section{Interaction between Parkin and the HCV Core Protein}

A loss of $\Delta \Psi$ stabilizes the mitochondrial accumulation of PINK1, and PINK1 recruits Parkin from the cytoplasm to depolarized mitochondria via its kinase activity. ${ }^{11-15} \mathrm{We}$ confirmed that Parkin was phosphorylated to the same degree after CCCP treatment regardless of $\mathrm{HCV}$ infection. Therefore, we next examined the mitochondrial accumulation of PINK1. Our results indicate that PINK1 accumulated in the mitochondrial fraction after CCCP treatment, and PINK1 expression levels in whole cell lysates were comparable irrespective of $\mathrm{HCV}$ infection (Figure 4A). In addition, blocking PINK1 protein expression with siRNA (Figure 4B) strikingly suppressed Parkin phosphorylation (Figure 4C) and the mitochondrial Parkin signal after CCCP treatment in Huh7 cells (Figure 4D), indicating that PINK1 recruits Parkin from the cytoplasm to depolarized mitochondria via its kinase activity. Suppressed translocation of Parkin to the mitochondria by HCV infection was also confirmed after treatment with valinomycin, a $\mathrm{K}^{+}$ionophore that rapidly dissipates $\Delta \Psi^{32}$ (Figure 4E).

We next examined the association between HCV protein and Parkin and hypothesized that HCV proteins may suppress Parkin translocation to the mitochondria. Coimmunoprecipitation experiments revealed that Parkin associated with the $\mathrm{HCV}$ core protein but not other $\mathrm{HCV}$ proteins, such as NS3, NS4A, and NS5A, regardless of CCCP treatment (Figure 5A). These results suggest that the $\mathrm{HCV}$ core protein specifically suppressed Parkin translocation to impaired mitochondria by interacting with Parkin.

Finally, we investigated which specific Parkin domain is critical for the interaction with the HCV core protein. The proposed Parkin architecture consists of an N-terminal ubiquitin-like domain, a really interesting new gene (RING) 0 domain (RING0), and a C-terminal in-between RING domain $^{33}$ (Figure 5B). Of these domains, the RING0 domain and a complete carboxy-terminal RING configuration are critical for the translocation of Parkin to damaged mitochondria and for consequent mitophagy. ${ }^{13}$ By using the $\mathrm{HCV}$ core protein as bait and either an $\mathrm{N}$-terminal fragment of Parkin, including RING0 (designated Parkin 1 to 215), or a C-terminal fragment of Parkin, not including RING0 (designated Parkin 216 to 465) as prey, a Yeast Two-Hybrid assay identified a specific interaction between Parkin 1 to 215 and the HCV core protein, which was visualized as a strong blue color (activation of the MELI gene encoding
A

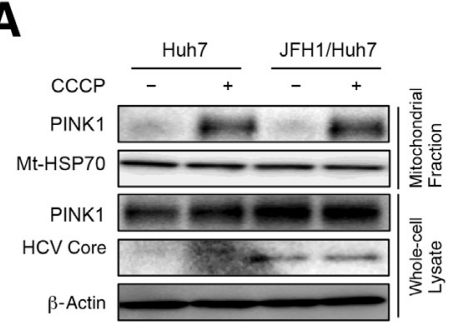

C

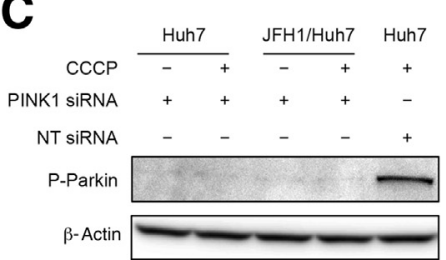

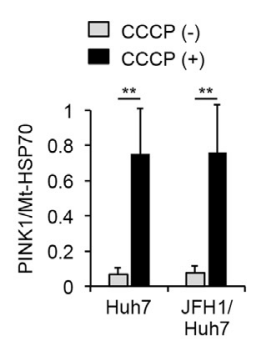

D

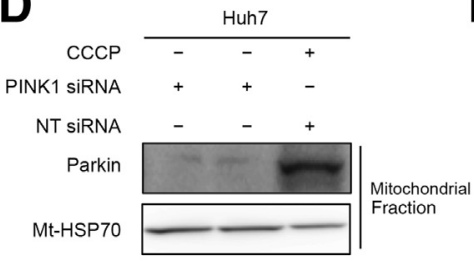

B

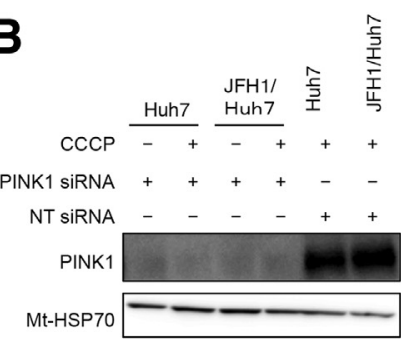

E

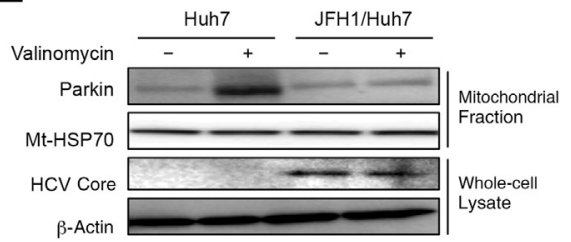

Figure 4 Mitochondrial accumulation of PINK1 after carbonyl cyanide $m$-chlorophenylhydrazone (CCCP) treatment and effect of PINK1 silencing on phosphorylation and mitochondrial translocation of Parkin. A: Immunoblots for PINK1 using mitochondrial fractions or whole cell lysates of Huh7 and JFH1Huh7 cells before and after CCCP treatment $(n=5)$. Immunoblots for PINK1 using mitochondrial fractions (B), for phosphorylated Parkin (P-Parkin) using whole cell lysates (C), and for Parkin using mitochondrial fractions (D) of Huh7 and/or JFH1-Huh7 cells before and after CCCP treatment with or without an siRNA-mediated blockade of PINK1 expression. E: Immunoblots for Parkin using the mitochondrial fractions of Huh7 and JFH1-Huh7 cells before and after a 3-hour valinomycin treatment. ${ }^{*} P<0.01$. Mt-HSP70, mitochondrial heat shock protein 70; NT siRNA, nontargeting siRNA. 
A

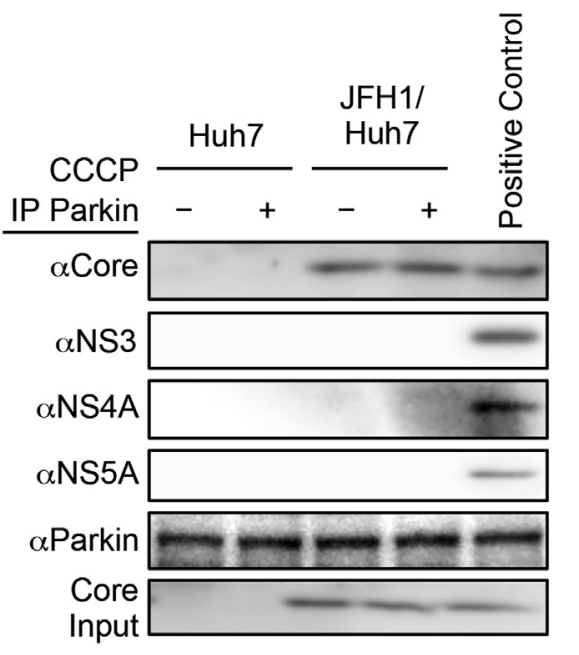

B

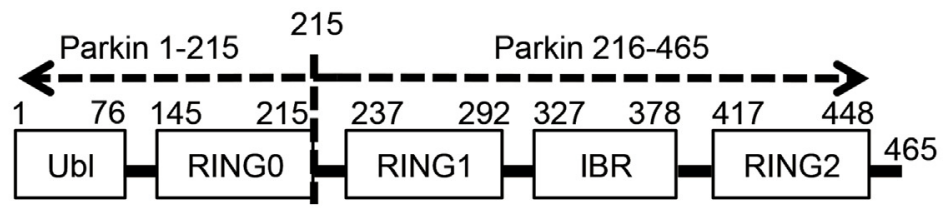

C

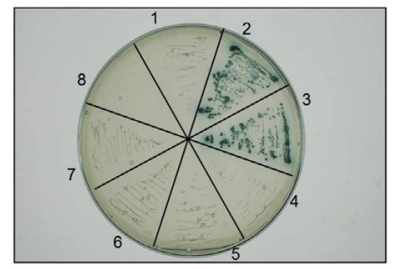

D

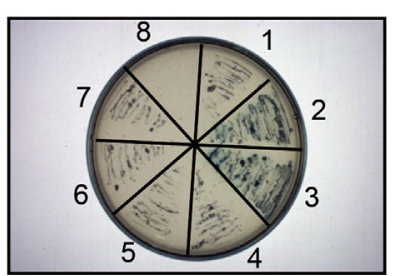

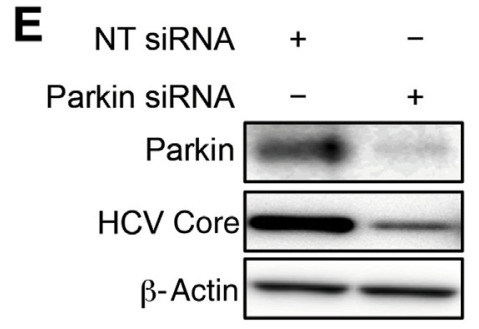

F

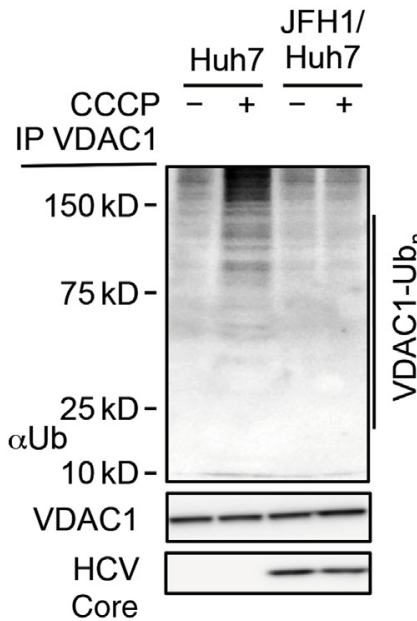

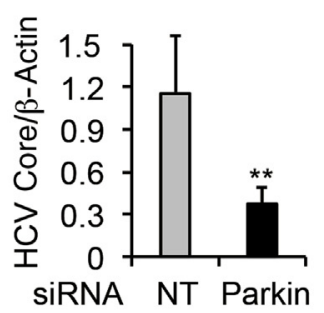

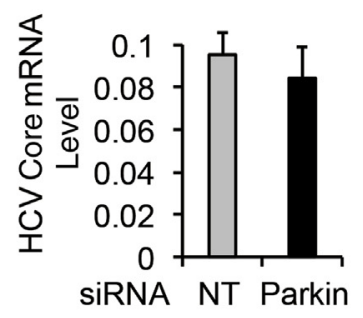

G

G JFH1/

Huh7 Huh7

JFH1/

CCCP $\frac{\text { Huh7 }}{-+} \frac{\text { Huh7 }}{-+}$

IP VDAC1

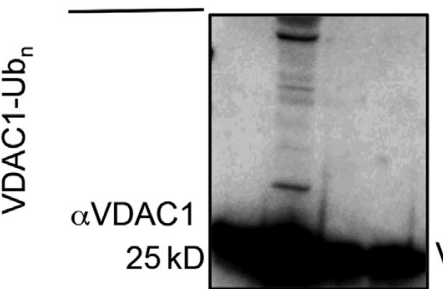

VDAC1

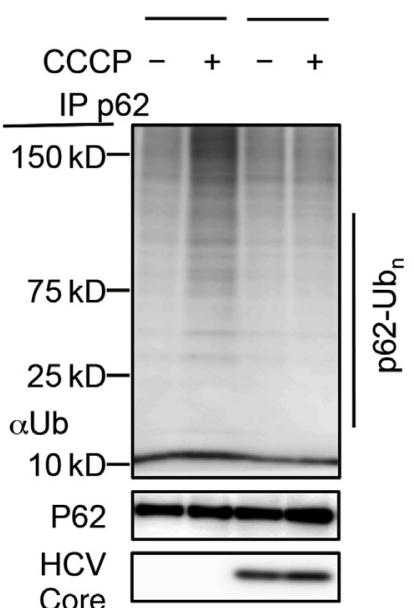

Figure 5 Interaction between Parkin and HCV core protein, effect of Parkin silencing on HCV replication, and reduction of mitochondrial outer membrane ubiquitination. A: Coimmunoprecipitation reveals a specific interaction of Parkin with the HCV core protein. B: The proposed Parkin architecture and a schematic diagram of Parkin domains. C and D: A Yeast Two-Hybrid assay identifies a specific interaction between Parkin 1 to 215 and the HCV core protein. The bait and prey for each section ( 1 to 8 ) in $\mathbf{C}$ are as follows: 1 , none and none; 2, p53 and large T antigen (positive control); 3, HCV core and Parkin 1 to 215; 4, HCV core and Parkin 216 to 465; 5, HCV core and none; 6, none and Parkin 1 to 215; 7, none and Parkin 216 to 465; 8, no yeast. The bait and prey for each section in C were reversed in D. E: The HCV core protein and HCV core mRNA levels in JFH1-Huh7 cells with or without an siRNA-mediated blockade of Parkin expression $(n=5)$. F: Coimmunoprecipitation reveals more VDAC1 ubiquitination in Huh7 cells after carbonyl cyanide m-chlorophenylhydrazone (CCCP) treatment. Various sizes of VDAC1 immunoprecipitates are also detected by immunoblotting using an anti-VDAC1 antibody in Huh7 cells after CCCP treatment. G: Coimmunoprecipitation reveals more p62 ubiquitination in Huh7 cells after CCCP treatment. ${ }^{*} P<0.01$. IBR, in-between RING; NT, nontargeting siRNA; Parkin, Parkin-targeting siRNA; Ubl, ubiquitin-like.

$\alpha$-galactosidase) (Figure 5C). In contrast, Parkin 216 to 465 did not interact with the HCV core protein. The same results were found when the core protein was used as prey and different domains of Parkin were used as bait (Figure 5D), indicating that this interaction between the two proteins was nonpolar. A previous mutational analysis of Parkin revealed that soluble Parkin mutants K211N, T240R, and G430D do not translocate to the mitochondria. ${ }^{13}$ Although we have not 

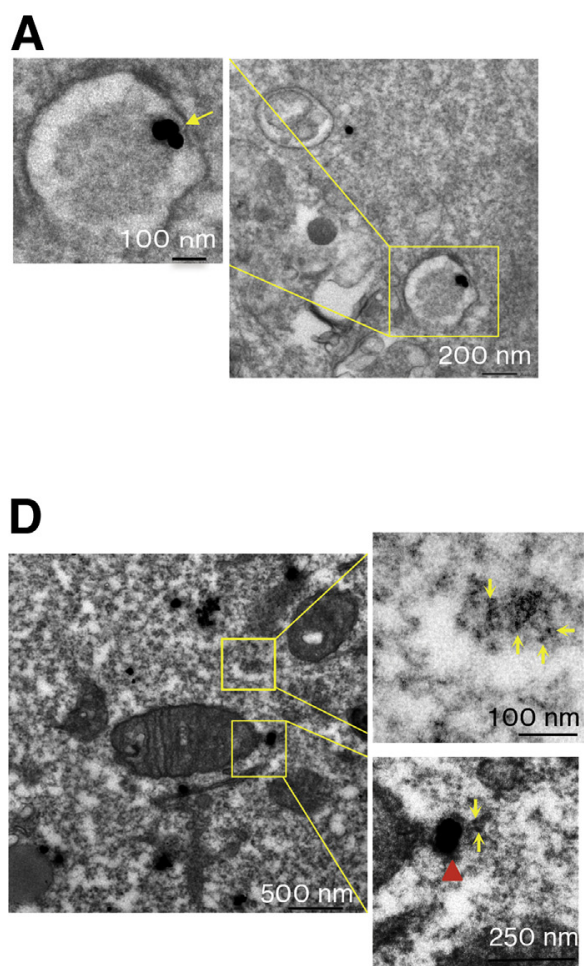

B

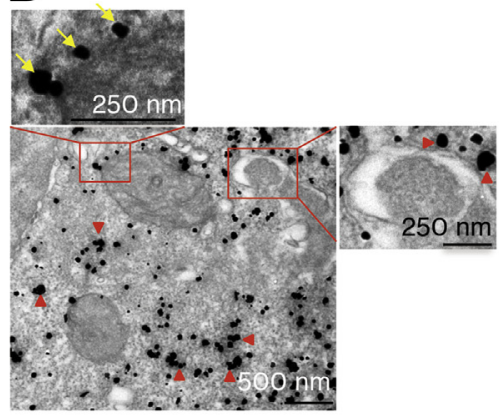

E

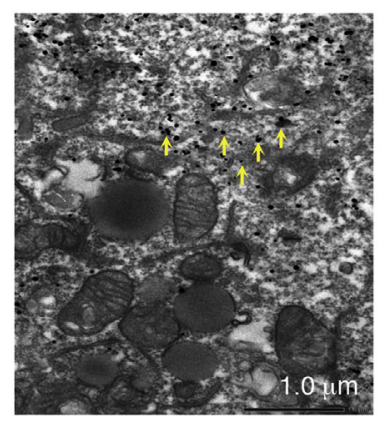

C

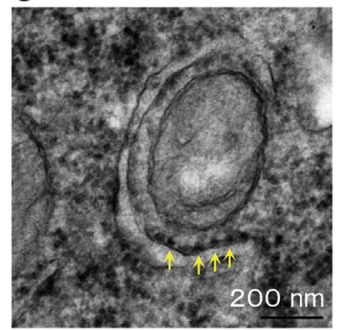

$\mathbf{F}$

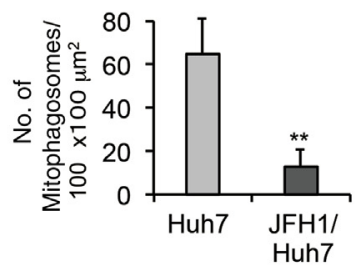

Figure 6 Electron microscopy of Huh7 cells and JFH1-Huh7 cells after carbonyl cyanide $m$-chlorophenylhydrazone (CCCP) treatment. A-E: Electron micrographs. Boxed areas are enlarged on left (Huh7 cell; A), above and on the right (Huh7 cell; B), and on the right (JFH1-Huh7 cell; D). The arrows indicate Parkin labeled with gold on the mitochondrial outer membrane (A and B), LC3 protein labeled with diaminobenzidine (DAB) on elongating isolation membrane that sequesters a single mitochondrion (Huh7 cells; C), Parkin core (D), and Parkin labeled with gold (JFH1-Huh7 cell; E). The arrowheads indicate Parkin labeled with gold (B) and HCV core (D). F: The number of mitophagosomes per $100 \times 100 \mu \mathrm{m}^{2}$ was calculated for four randomly selected views. ${ }^{* *} P<0.01$.

determined whether the HCV core protein binds to the region that includes lysine (K) 211 in the RING0 domain, the specific interaction of Parkin 1 to 215 with the HCV core protein raises the possibility that the core protein inhibits Parkin translocation to the mitochondria by affecting lysine 211.

After we confirmed the specific interaction between the HCV core protein and Parkin, we investigated whether Parkin affects HCV replication to investigate the functional role of the interaction between both proteins in the HCV infectious process. Parkin silencing significantly inhibited $\mathrm{HCV}$ replication, as indicated by a decrease in HCV core protein expression, but did not affect $\mathrm{HCV}$ core mRNA levels (Figure 5E). These results suggest that the association of the HCV core protein with Parkin plays a functional role in $\mathrm{HCV}$ propagation, although further studies are required to clarify the mechanisms.

\section{Suppressed Ubiquitination of the Mitochondrial Outer Membrane Protein VDAC1}

The next step in mitophagy after Parkin translocation to the mitochondria is the ubiquitination of mitochondrial outer membrane proteins. ${ }^{13,16}$ Coimmunoprecipitation experiments revealed that various sizes of ubiquitinated VDAC1 species in the mitochondrial outer membrane ${ }^{13}$ were present after CCCP treatment in Huh7 cells but not in JFH1-Huh7 cells (Figure 5F). Western blot analysis of VDAC1 immunoprecipitates revealed various sizes of VDAC1 species after CCCP treatment in Huh7 cells but not in JFH1-Huh7 cells (Figure 5F). The autophagic adaptor p62 aggregates ubiquitinated proteins by polymerizing with other p62 molecules. ${ }^{13}$ Similarly, coimmunoprecipitation experiments revealed that CCCP treatment induced various sizes of ubiquitinated p62 species in Huh7 cells but not in JFH1Huh7 cells (Figure 5G). These results suggest that HCV infection inhibited the Parkin-induced ubiquitination of the depolarized mitochondria.

\section{Suppressed Mitophagosome Formation}

During mitophagy, the isolation membrane sequesters a single mitochondrion or a cluster of mitochondria to form an autophagosome (mitophagosome). A single mitochondrion with Parkin on its outer membrane was sequestered by the isolation membrane after CCCP treatment in Huh7 cells (Figure 6A). Parkin in close proximity to the mitochondria and association of Parkin with mitochondrial outer membrane were observed more frequently in Huh7 cells than in JFH1-Huh7 cells (Figure 6, B, D, and E). In addition, LC3 was present on elongating isolation membrane that sequesters a single mitochondrion after CCCP treatment in Huh7 cells (Figure 6C). The number of mitophagosomes, calculated as the number of autophagosomes that contain 
A

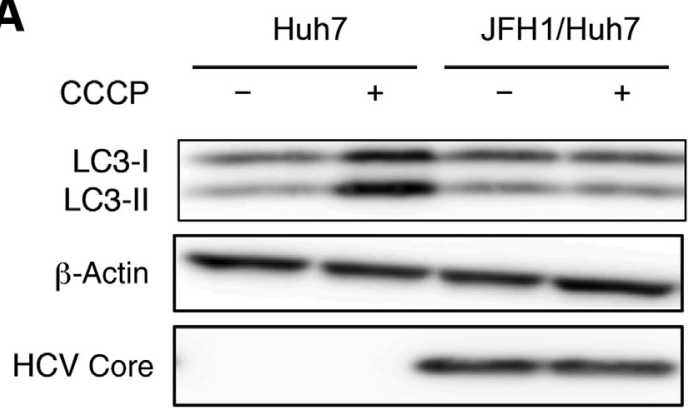

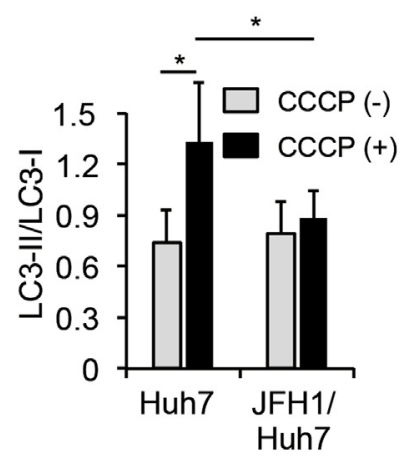

B

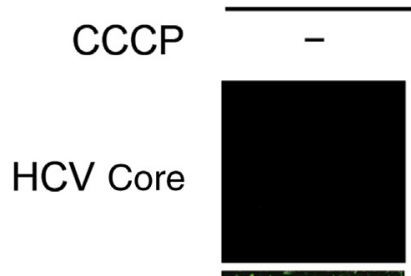

Huh7
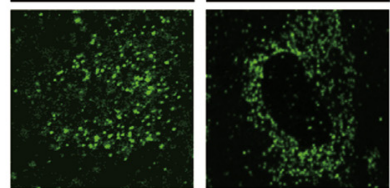

Parkin
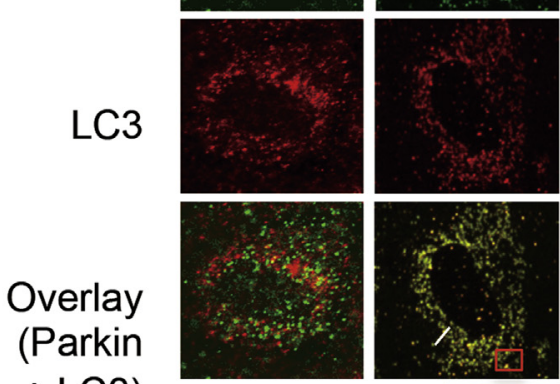

+ LC3)
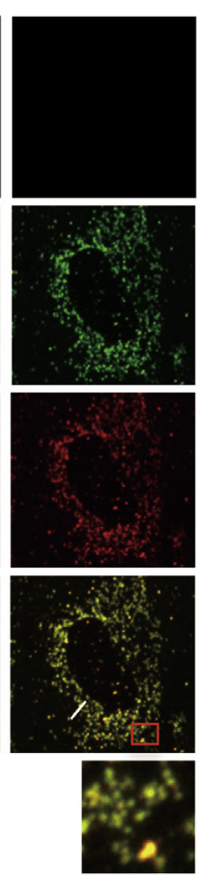
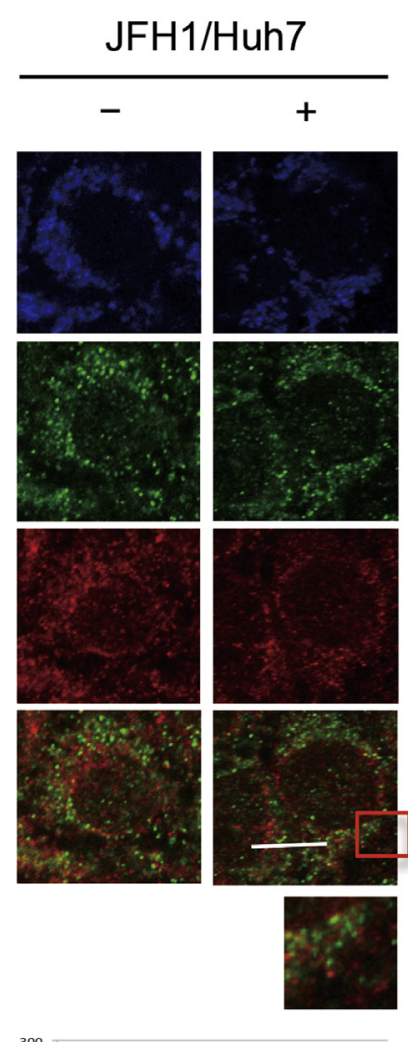

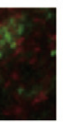

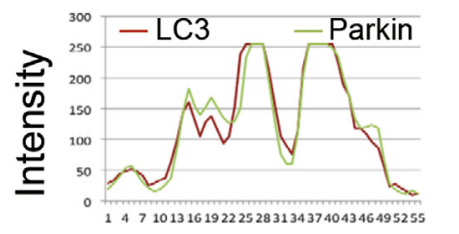

Distance $(\mu \mathrm{m})$

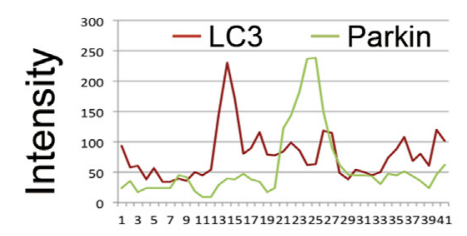

Distance $(\mu \mathrm{m})$
Figure 7 Effect of HCV infection on LC3-II expression and colocalization of Parkin with LC3 after carbonyl cyanide $m$-chlorophenylhydrazone (CCCP) treatment. A: Immunoblots for LC3-I and LC3-II using whole cell lysates of Huh7 and JFH1Huh7 cells before and after CCCP treatment $(n=5)$. B: Immunofluorescence staining for Parkin (green) and LC3 (red) in Huh7 and JFH1-Huh7 cells before and after a 1-hour CCCP treatment. Boxed areas are enlarged below. Endogenous Parkin that colocalizes with LC3 (yellow spots). Line scans indicate the colocalization of Parkin with LC3 and correlate to the white lines in the images. C: The expression of LC3 mRNA in the liver from nontransgenic (non-TgM) and $\operatorname{TgM}$ mice $(n=5)$ and from chimeric mice without or with HCV infection $(n=5)$. The expression level of LC3 mRNA was normalized to GAPDH. ${ }^{*} P<0.05$.

\section{C}

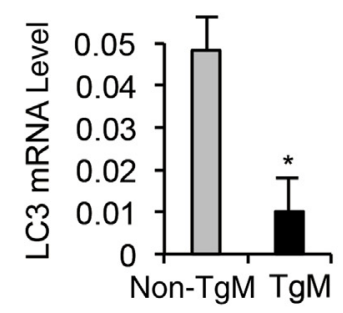

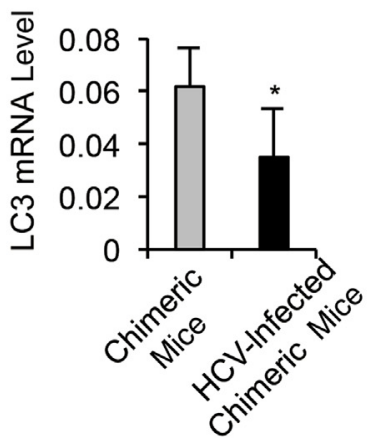


mitochondria, was significantly reduced in JFH1-Huh7 cells compared with Huh7 cells (Figure 6F). Therefore, HCV infection clearly suppressed mitophagosome formation.

In agreement with suppressed mitophagosome formation, the LC3-II/I ratio was significantly lower after CCCP treatment in JFH1-Huh7 cells compared with Huh7 cells (Figure 7A), although the LC3-II/I ratio itself increased after CCCP treatment regardless of $\mathrm{HCV}$ infection. LC3 has been shown to be present in both complete autophagosomes and elongating isolation membranes that contain mitochondria ubiquitinated by Parkin. The present results indicate that Parkin colocalized with LC3 after CCCP treatment in Huh7 cells, whereas colocalization of Parkin and LC3 was significantly reduced in JFH1-Huh7 cells (Figure 7B). In vivo, FL-N/35-transgenic mice and $\mathrm{HCV}$-infected chimeric mice also showed significantly reduced expression levels of LC3 mRNA in the liver compared with the control mice (Figure 7C), in agreement with reduced expression of Parkin in the mitochondrial fraction. These results may seem to be inconsistent with increased protein level of LC3-II after CCCP treatment in vitro. However, the lower LC3-II/I ratio after CCCP treatment in HCV-infected cells than in noninfected cells may reflect reduced expression levels of LC3 mRNA in FL-N/35-transgenic mice and $\mathrm{HCV}$-infected chimeric mice. Further studies are required to clarify the mechanisms.

Several previous studies have proposed that autophagosome accumulation is enhanced on HCV infection and in HCV replicon cell lines. ${ }^{34-38}$ Our findings of a decreased LC3-II/I ratio in JFH1-Huh7 cells, FL-N/35-transgenic mice, and $\mathrm{HCV}$-infected chimeric mice seemingly contradict these previous reports. To clarify whether the decrease in LC3-II/I ratio observed in the present study indicated that macroautophagy (generally referred to as autophagy) or mitophagy was inhibited, we investigated LC3-II/I ratio in JFH1-Huh7 and Huh7 cells using Earle's balanced salt solution (EBSS) as a macroautophagy inducer (via amino acid starvation). ${ }^{39}$ Interestingly, JFH1-Huh7 cells showed significantly increased LC3-II/I ratio compared with Huh7 cells after incubation with EBSS for 1 hour (Figure 8A), suggesting that $\mathrm{HCV}$ infection promoted autophagy under macroautophagy-inducible conditions. In agreement with increased LC3-II/I ratio, electron microscopy revealed that the number of autophagosomes was significantly greater after EBSS treatment in JFH1-Huh7 cells than in Huh7 cells (Figure 8B). Taken together with these results, the decrease in LC3-II/I ratio observed after CCCP treatment in JFH1-Huh7 cells likely represents a consequence of mitophagy inhibition, but not autophagy inhibition by $\mathrm{HCV}$ infection.

\section{Suppression of Autophagic Degradation}

The autophagic adaptor p62 can both aggregate ubiquitinated proteins by polymerizing with other p62 molecules and recruit ubiquitinated cargo into mitophagosomes by

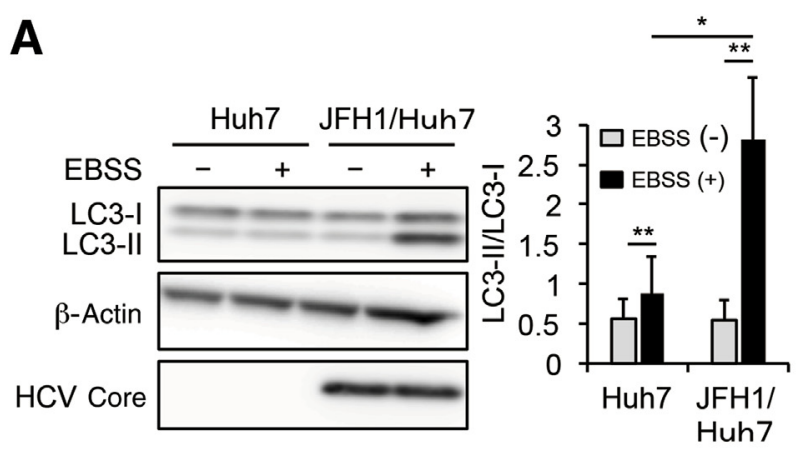

B

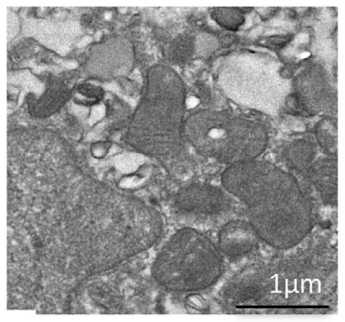

C

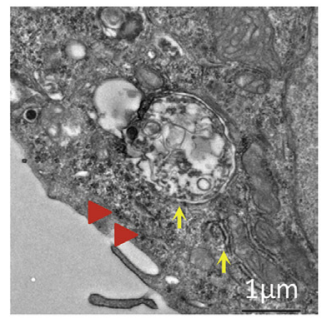

D

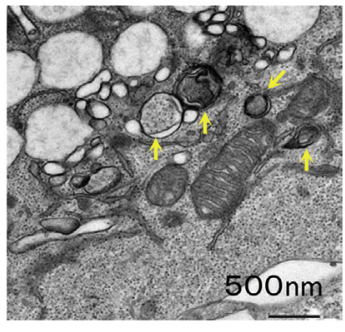

E

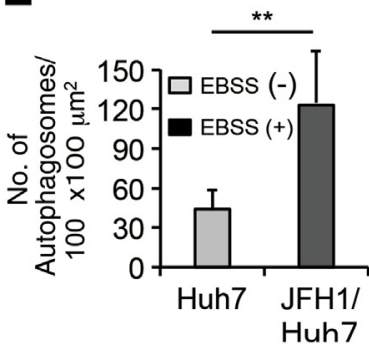

Figure 8 Effect of HCV infection on LC3-II expression and autophagosome formation after culture with Earle's balanced salt solution (EBSS). A: Immunoblots for LC3-II using Huh7 and JFH1-Huh7 cells before (-) and after $(+)$ culture with EBSS $(n=6)$. The LC3-II and LC3-I expression level was normalized to $\beta$-actin. Electron microscopy of Huh7 (B) and JFH1-Huh7 (C and D) cells after EBSS treatment. The arrows indicate autophagosomes; arrowheads, HCV core protein. E: The number of autophagosomes per $100 \times 100 \mu \mathrm{m}^{2}$ was calculated for five randomly selected views. ${ }^{*} P<0.05$, ${ }^{*} P<0.01$.

binding to LC3-II. ${ }^{13}$ Therefore, p62 accumulation can be attributed to a deficit in autophagic degradation activity. After a 1- or 2-hour CCCP treatment, there was a smaller decrease in p62 in JFH1-Huh7 cells compared with Huh7 cells (Figure 9A). In vivo, FL-N/35-transgenic mice and $\mathrm{HCV}$-infected chimeric mice also showed p62 accumulation in the liver compared with the control mice (Figure 9B). These results suggest that the degradation of damaged mitochondria was suppressed in the presence of $\mathrm{HCV}$ infection.

Finally, we assessed the change in VDAC1 content after CCCP treatment to obtain additional evidence as to whether mitophagy itself was suppressed by HCV infection. After a 2-hour CCCP treatment, a decrease in cellular content of VDAC1 was significantly smaller in JFH1-Huh7 cells than in Huh7 cells (Figure 9C). We also found that CCCP-induced increase in ROS production was greater in 


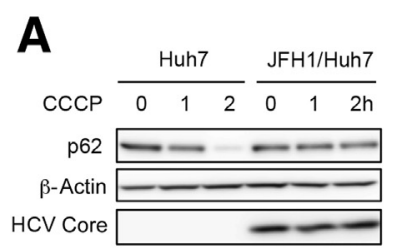

B

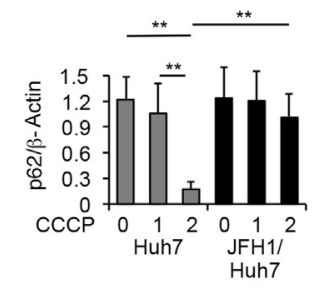

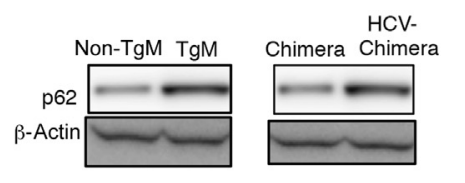

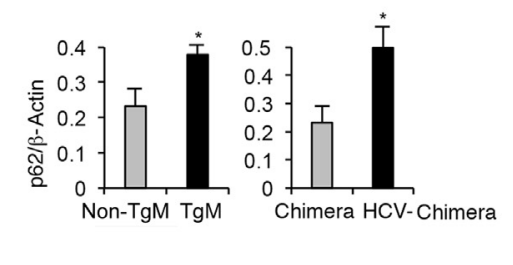

C
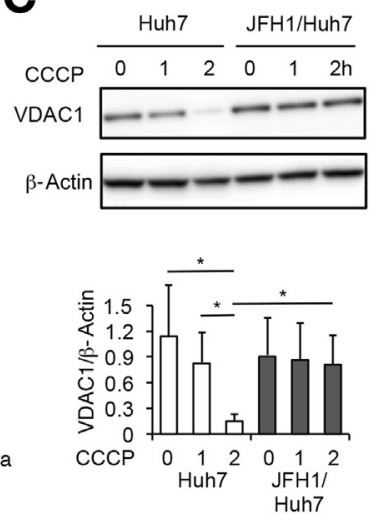

D

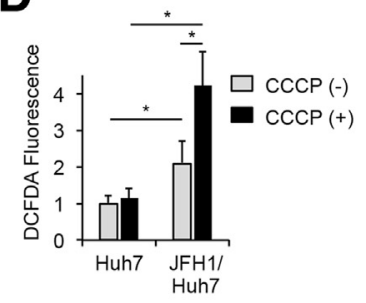

Figure 9 Effect of HCV infection on cellular p62 and VDAC1 expression after carbonyl cyanide $m$-chlorophenylhydrazone (CCCP) treatment and reactive oxygen species (ROS) production. A: Immunoblots for p62 using whole cell lysates of Huh7 and JFH1-Huh7 cells before and after a 1- and a 2-hour CCCP treatment $(n=5)$. B: Immunoblots for p62 using the liver from non-transgenic (non- $\operatorname{TgM})$ and $\operatorname{TgM}(n=5)$ mice and from chimeric mice without or with HCV infection $(n=5)$. The p62 expression level was normalized to $\beta$-actin. C: Immunoblots for VDAC1 using whole cell lysates of Huh7 and JFH1-Huh7 cells before and after a 1- and a 2-hour CCCP treatment $(n=5)$. The VDAC1 expression level was normalized to $\beta$-actin. D: Changes in cellular ROS production after a 1-hour CCCP treatment in Huh7 and JFH1-Huh7 cells $(n=5) .{ }^{*} P<0.05,{ }^{*} P<0.01$.

JFH1-Huh7 cells than in Huh7 cells (Figure 8D). These results were consistent with a previous study that showed an essential role of mitophagy in reducing mitochondrial ROS production $^{40}$ and, therefore, may reflect the suppressed mitophagy in the presence of $\mathrm{HCV}$ infection.

\section{Discussion}

Mitophagy may likely be induced in HCV-JFH1-infected cells in the context of mitochondrial depolarization, and in transgenic mice expressing the $\mathrm{HCV}$ polyprotein or in HCV-infected chimeric mice, both of which showed the decreased mitochondrial GSH content. Our results suggest that the $\mathrm{HCV}$ core protein inhibits mitophagy during $\mathrm{HCV}$ infection and that the molecular mechanisms by which this suppression occurs include the interaction of the HCV core protein with Parkin and the inhibition of Parkin translocation to the mitochondria. This inhibition leads to the failure of mitochondrial ubiquitination, mitophagosome formation, and autophagic degradation (Figure 10). Because
Parkin 1 to 215 contains one of the critical amino acids required for mitochondrial localization, the specific interaction of Parkin 1 to 215 with the $\mathrm{HCV}$ core protein strongly suggests that the core protein represses mitophagy by inhibiting Parkin translocation to the mitochondria. We know that PINK1 accumulates in the mitochondria and phosphorylates Parkin after CCCP treatment and that the suppression of the mitochondrial Parkin signal occurs by blocking PINK1 via siRNA. Therefore, we could exclude the possibility that PINK1 plays a role in suppressing the recruitment of Parkin to the mitochondria. To our knowledge, this is the first report to demonstrate a suppressive effect of a viral protein on mitophagy via an interaction with Parkin. Interestingly, silencing Parkin via siRNA inhibited $\mathrm{HCV}$ core expression, which was consistent with the results of a recent study. ${ }^{28}$ These results suggest that HCV potentially uses Parkin for its replication through the interaction between the HCV core protein and Parkin. Parkin may be post-transcriptionally involved in $\mathrm{HCV}$ replication, because Parkin silencing did not affect HCV core mRNA levels.

\section{Suppression of Recruitment of Parkin to Mitochondria}

Suppression of Mitophagosomal Formation
Suppression of Autophagic Degradation

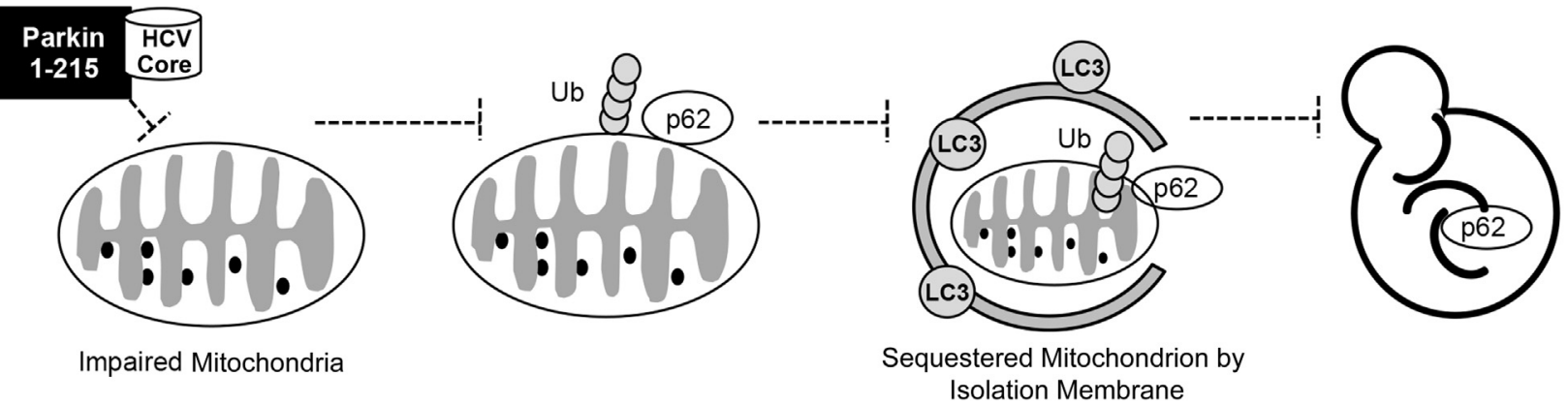

Figure 10 A schematic diagram depicting the mechanisms underlying mitophagy suppression by the HCV core protein. The HCV core protein interacts with the Parkin N-terminal fragment containing the RINGO domain (designated Parkin 1 to 215) and inhibits Parkin translocation to the mitochondria, which leads to the failure of mitochondrial ubiquitination, autophagosome formation, and autophagic degradation. Ub, ubiquitin. 
Further studies are required to clarify the mechanisms underlying this speculation.

Two types of autophagy have been identified: nonselective and selective. For nonselective autophagy related to $\mathrm{HCV}$ infection, previous studies have reported the enhanced accumulation of autophagosomes without any effect on autophagic protein degradation, ${ }^{33}$ the requirement of LC3 for efficient $\mathrm{HCV}$ replication, ${ }^{34}$ and the occurrence of HCV RNA replication on autophagosomal membranes. ${ }^{36}$ Mitophagy is selective and is induced by mitochondrial membrane depolarization, followed by Parkin recruitment to the mitochondria. ${ }^{9-15}$ Herein, mitophagosome accumulation was suppressed because of mitophagy inhibition, whereas HCV infection enhanced the expression of LC3-II and autophagosome accumulation under nonselective autophagy-inducible conditions. Therefore, the present results are consistent with the previously characterized HCVinduced nonselective autophagic response. ${ }^{34,35,38}$ However, a recent report has shown that HCV induces the mitochondrial translocation of Parkin and subsequent mitophagy, ${ }^{28}$ which contrasts with the present results, except for the inhibitory effect of Parkin silencing on HCV replication. One of the significant differences in the method between the two studies was the presence or absence of CCCP treatment. Whether HCV-induced mitophagy was preceded by mitochondrial depolarization was unknown because $\Delta \Psi$ was not measured in the previous report of HCV-induced mitophagy. ${ }^{28}$ However, we need to be careful that the mitochondrial depolarization by CCCP treatment is not a pathophysiological condition observed in $\mathrm{HCV}$ infection and that CCCP causes the depolarization of the entire mitochondrial network. ${ }^{41}$ It is currently unknown whether CCCP treatment caused paradoxical results on mitophagy in $\mathrm{HCV}$-infected cells between our study and a previous one. ${ }^{28}$ Although suppressed mitophagy was also found in FL-N/ 35-transgenic mice and HCV-infected chimeric mice without any treatment, these mice may not be simply compared with HCV-JFH1-infected cells in terms of extremely low levels of viral proteins in FL-N/35-transgenic mice or spontaneous oxidized mitochondrial glutathione in both mice. Another difference between two studies was postinfection time from infection to assessment of mitophagy in HCV-JFH1-infected cells (21 versus 3 days). However, further studies are required to clarify whether postinfection time of HCV-JFH1-infected cells affects the interaction of $\mathrm{HCV}$ with Parkin. Oxidative stress and/or hepatocellular mitochondrial alterations are present in chronic hepatitis $\mathrm{C}$ to a greater degree than in other inflammatory liver diseases, ${ }^{1,6}$ and mitophagy is important for maintaining mitochondrial quality by eliminating damaged mitochondria. Therefore, our results that the HCV core protein suppresses mitophagy appear reasonable in the context of what is known about the pathophysiological characteristics of chronic hepatitis $\mathrm{C}$.

$\mathrm{HCV}$-induced mitochondrial injury, ROS production, and subsequent oxidative stress contribute to HCC development in FL-N/35-transgenic mice that receive modest iron supplementation. ${ }^{8}$ The relatively long period (12 months) required for $\mathrm{HCC}$ development suggests that mitochondrial injury, as a source of oxidative stress, must continue for a prolonged period. Mitochondrial DNA mutations are also relevant to $\mathrm{HCC}$ development in patients with chronic $\mathrm{HCV}$ infections. ${ }^{42}$ Indeed, mitophagy plays an essential role in reducing mitochondrial ROS production and mitochondrial DNA mutations in yeast ${ }^{40}$ and eliminating oxidative damaged mitochondria. ${ }^{43}$ In addition to the directly induced generation of ROS by HCV proteins, the suppression of mitophagy by the $\mathrm{HCV}$ core protein has the potential to generate an additional long-lasting ROS burden and may offset or overwhelm the physiological antioxidative activity in mitochondria. Therefore, the suppressive effect of the $\mathrm{HCV}$ core protein on mitophagy may be an important mechanism of $\mathrm{HCV}$-induced hepatocarcinogenesis.

In conclusion, results indicate that $\mathrm{HCV}$ core protein suppresses mitophagy by inhibiting Parkin translocation to the mitochondria via a direct interaction with Parkin in the context of mitochondrial depolarization. These findings have implications for the amplification and sustainability of mitochondria-induced oxidative stress observed in patients with HCV-related chronic liver disease and an increased risk of hepatocarcinogenesis.

\section{Acknowledgments}

We thank Dr. Stanley M. Lemon for the transgenic mice, Dr. Takaji Wakita for the JFH1 clone, and Hikari Hara for technical assistance.

\section{References}

1. Farinati F, Cardin R, De Maria N, Della Libera G, Marafin C, Lecis E, Burra P, Floreani A, Cecchetto A, Naccarato R: Iron storage, lipid peroxidation and glutathione turnover in chronic anti-HCV positive hepatitis. J Hepatol 1995, 22:449-456

2. Valgimigli M, Valgimigli L, Trere D, Gaiani S, Pedulli GF, Gramantieri L, Bolondi L: Oxidative stress EPR measurement in human liver by radical-probe technique: correlation with etiology, histology and cell proliferation. Free Radic Res 2002, 36:939-948

3. Okuda M, Li K, Beard MR, Showalter LA, Scholle F, Lemon SM Weinman SA: Mitochondrial injury, oxidative stress, and antioxidant gene expression are induced by hepatitis $\mathrm{C}$ virus core protein. Gastroenterology 2002, 122:366-375

4. Moriya K, Nakagawa K, Santa T, Shintani Y, Fujie H, Miyoshi H, Tsutsumi T, Miyazawa T, Ishibashi K, Horie T, Imai K, Todoroki T, Kimura S, Koike K: Oxidative stress in the absence of inflammation in a mouse model for hepatitis $\mathrm{C}$ virus-associated hepatocarcinogenesis. Cancer Res 2001, 61:4365-4370

5. Korenaga M, Wang T, Li Y, Showalter LA, Chan T, Sun J, Weinman SA: Hepatitis C virus core protein inhibits mitochondrial electron transport and increases reactive oxygen species (ROS) production. J Biol Chem 2005, 280:37481-37488

6. Barbaro G, Di Lorenzo G, Asti A, Ribersani M, Belloni G, Grisorio B, Filice G, Barbarini G: Hepatocellular mitochondrial alterations in patients with chronic hepatitis C: ultrastructural and biochemical findings. Am J Gastroenterol 1999, 94:2198-2205 
7. Nishina S, Hino K, Korenaga M, Vecchi C, Pietrangelo A, Mizukami Y, Furutani T, Sakai A, Okuda M, Hidaka I, Okita K, Sakaida I: Hepatitis C virus-induced reactive oxygen species raise hepatic iron level in mice by reducing hepcidin transcription. Gastroenterology 2008, 134:226-238

8. Furutani T, Hino K, Okuda M, Gondo T, Nishina S, Kitase A, Korenaga M, Xiao SY, Weinman SA, Lemon SM, Sakaida I, Okita K: Hepatic iron overload induces hepatocellular carcinoma in transgenic mice expressing the hepatitis C virus polyprotein. Gastroenterology 2006, 130:2087-2098

9. Kim I, Rodriguez-Enriquez S, Lemasters JJ: Selective degradation of mitochondria by mitophagy. Arch Biochem Biophys 2007, 462:245-253

10. Elmore SP, Qian T, Grissom S, Lemasters JJ: The mitochondrial permeability transition initiates autophagy in rat hepatocytes. FASEB J 2001, 15:2286-2287

11. Matsuda N, Sato S, Shiba K, Okatsu K, Saisho K, Gautier CA, Sou YS, Saiki S, Kawajiri S, Sato F, Kimura M, Komatsu M, Hattori N, Tanaka K: PINK1 stabilized by mitochondrial depolarization recruits Parkin to damaged mitochondria and activates latent Parkin for mitophagy. J Cell Biol 2010, 189:211-221

12. Narendra DP, Jin SM, Tanaka A, Suen DF, Gautier CA, Shen J, Cookson MR, Youle RJ: PINK1 is selectively stabilized on impaired mitochondria to activate Parkin. PLoS Biol 2010, 8:e1000298

13. Geisler S, Holmström KM, Skujat D, Fiesel FC, Rothfuss OC, Kahle PJ, Springer W: PINK1/Parkin-mediated mitophagy is dependent on VDAC1 and p62/SQSTM1. Nat Cell Biol 2010, 12:119-131

14. Vives-Bauza C, Zhou C, Huang Y, Cui M, de Vries RL, Kim J, May J, Tocilescu MA, Liu W, Ko HS, Magrane J, Moore DJ, Dawson VL, Grailhe R, Dawson TM, Li C, Tieu K, Przedborski S: PINK1-dependent recruitment of Parkin to mitochondria in mitophagy. Proc Natl Acad Sci USA 2010, 107:378-383

15. Narendra D, Tanaka A, Suen DF, Youle RJ: Parkin is recruited selectively to impaired mitochondria and promotes their autophagy. J Cell Biol 2008, 183:795-803

16. Chan NC, Salazar AM, Pham AH, Sweredoski MJ, Kolawa NJ, Graham RL, Hess S, Chan DC: Broad activation of the ubiquitinproteasome system by Parkin is critical for mitophagy. Hum Mol Genet 2011, 20:1726-1737

17. Gegg ME, Cooper JM, Chau KY, Rojo M, Schapira AH, Taanman JW: Mitofusin 1 and mitofusin 2 are ubiquitinated in a PINK1/parkindependent manner upon induction of mitophagy. Hum Mol Genet 2010, 19:4861-4870

18. Chen D, Gao F, Li B, Wang H, Xu Y, Zhu C, Wang G: Parkin monoubiquitinates Bcl-2 and regulates autophagy. J Biol Chem 2010, 285: 38214-38223

19. Narendra D, Kane LA, Hauser DN, Fearnley IM, Youle RJ: p62/SQSTM1 is required for Parkin-induced mitochondrial clustering but not mitophagy: VDAC1 is dispensable for both. Autophagy 2010, 6:1090-1106

20. Itakura E, Kishi-Itakura C, Koyama-Honda I, Mizushima N: Structures containing Atg9A and the ULK1 complex independently target depolarized mitochondria at initial stages of Parkin-mediated mitophagy. J Cell Sci 2012, 125:1488-1499

21. Okatsu K, Saisho K, Shimanuki M, Nakada K, Shitara H, Sou YS, Kimura M, Sato S, Hattori N, Komatsu M, Tanaka K, Matsuda N: p62/SQSTM1 cooperates with Parkin for perinuclear clustering of depolarized mitochondria. Genes Cells 2010, 15:887-900

22. Wakita T, Pietschmann T, Kato T, Date T, Miyamoto M, Zhao Z, Murthy K, Habermann A, Kräusslich HG, Mizokami M, Bartenschlager R, Liang TJ: Production of infectious hepatitis $\mathrm{C}$ virus in tissue culture from a cloned viral genome. Nat Med 2005, 11:791-796

23. Li K, Prow T, Lemon SM, Beard MR: Cellular response to conditional expression of hepatitis $\mathrm{C}$ virus core protein in Huh7 cultured human hepatoma cells. Hepatology 2002, 35:1237-1246

24. Lerat H, Honda M, Beard MR, Loesch K, Sun J, Yang Y, Okuda M, Gosert R, Xiao SY, Weinman SA, Lemon SM: Steatosis and liver cancer in transgenic mice expressing the structural and nonstructural proteins of hepatitis C virus. Gastroenterology 2002, 122:352-365
25. Tateno C, Yoshizane Y, Saito N, Kataoka M, Utoh R, Yamasaki C, Tachibana A, Soeno Y, Asahina K, Hino H, Asahara T, Yokoi T, Furukawa T, Yoshizato K: Near completely humanized liver in mice shows human-type metabolic responses to drugs. Am J Pathol 2004, 165:901-912

26. Kimura T, Imamura M, Hiraga N, Hatakeyama T, Miki D, Noguchi C, Mori N, Tsuge M, Takahashi S, Fujimoto Y, Iwao E, Ochi H, Abe H, Maekawa T, Arataki K, Tateno C, Yoshizato K, Wakita T, Okamoto T, Matsuura Y, Chayama K: Establishment of an infectious genotype 1b hepatitis $\mathrm{C}$ virus clone in human hepatocyte chimeric mice. J Gen Virol 2008, 89:2108-2113

27. Ando M, Korenaga M, Hino K, Ikeda M, Kato N, Nishina S, Hidaka I, Sakaida I: Mitochondrial electron transport inhibition in full genomic hepatitis $\mathrm{C}$ replicon cells is restored by reducing viral replication. Liver Int 2008, 28:1158-1166

28. Kim SJ, Syed GH, Siddiqui A: Hepatitis C virus induces the mitochondrial translocation of Parkin and subsequent mitophagy. PLoS Pathog 2013, 9:e1003285

29. Toida K, Kosaka K, Aika Y, Kosaka T: Chemically defined neuron groups and their subpopulations in the glomerular layer of the rat main olfactory bulb, IV: intraglomerular synapses of tyrosine hydroxylaseimmunoreactive neurons. Neuroscience 2000, 101:11-17

30. Ikeda M, Sugiyama K, Mizutani T, Tanaka T, Tanaka K, Sekihara H, Shimotohno K, Kato N: Human hepatocyte clonal cell lines that support persistent replication of hepatitis C virus. Virus Res 1998, 56: $157-167$

31. Zhang GJ, Liu HW, Yang L, Zhong YG, Zheng YZ: Influence of membrane physical state on the lysosomal proton permeability. J Membr Biol 2000, 175:53-62

32. Sharpe MA, Wrigglesworth JM, Loewen J, Nicholls P: Small pH gradients inhibit cytochrome c oxidase: implications for $\mathrm{H}+$ entry to the binuclear center. Biochem Biophys Res Commun 1995, 216: 931-938

33. Hristova VA, Beasley SA, Rylett RJ, Shaw GS: Identification of a novel $\mathrm{Zn} 2+$-binding domain in the autosomal recessive juvenile Parkinsonrelated E3 ligase parkin. J Biol Chem 2009, 284:14978-14986

34. Sir D, Chen WL, Choi J, Wakita T, Yen TS, Ou JH: Induction of incomplete autophagic response by hepatitis $\mathrm{C}$ virus via the unfolded protein response. Hepatology 2008, 48:1054-1061

35. Dreux M, Gastaminza P, Wieland SF, Chisari FV: The autophagy machinery is required to initiate hepatitis $\mathrm{C}$ virus replication. Proc Natl Acad Sci U S A 2009, 106:14046-14051

36. Ke PY, Chen SS: Activation of the unfolded protein response and autophagy after hepatitis $\mathrm{C}$ virus infection suppresses innate antiviral immunity in vitro. J Clin Invest 2011, 121:37-56

37. Sir D, Kuo CF, Tian Y, Liu HM, Huang EJ, Jung JU, Machida K, Ou JH: Replication of hepatitis C virus RNA on autophagosomal membranes. J Biol Chem 2012, 287:18036-18043

38. Shrivastava S, Bhanja Chowdhury J, Steele R, Ray R, Ray RB: Hepatitis C virus upregulates Beclin1 for induction of autophagy and activates mTOR signaling. J Virol 2012, 86:8705-8712

39. Munafo DB, Colombo MI: A novel assay to study autophagy: regulation of autophagosome vacuole size by amino acid deprivation. J Cell Sci 2001, 114:3619-3629

40. Kurihara Y, Kanki T, Aoki Y, Hirota Y, Saigusa T, Uchiumi T, Kang D: Mitophagy plays an essential role in reducing mitochondrial production of reactive oxygen species and mutation of mitochondrial DNA by maintaining mitochondrial quantity and quality in yeast. J Biol Chem 2012, 287:3265-3272

41. Wang Y, Nartiss Y, Steipe B, McQibban GA, Kim PK: ROS-induced mitochondrial depolarization initiates PARK2/PARKIN-dependent mitochondrial degradation by autophagy. Autophagy 2012, 8:1462-1476

42. Nishikawa M, Nishiguchi S, Shiomi S, Tamori A, Koh N, Takeda T, Kubo S, Hirohashi K, Kinoshita H, Sato E, Inoue M: Somatic mutation of mitochondrial DNA in cancerous and noncancerous liver tissue in individuals with hepatocellular carcinoma. Cancer Res 2001, 61:1843-1845

43. Venditti P, Di Stefano L, Di Meo S: Mitochondrial metabolism of reactive oxygen species. Mitochondrion 2013, 13:71-82 